\title{
MICROESTRUTURA POROSA DE COQUE: RELAÇÃO COM A RESISTÊNCIA MECÂNICA*
}

\author{
Anderson Azevedo Agra ${ }^{1}$ \\ Anderson $\mathrm{Nicolodi}^{2}$ \\ Bruno Deves Flores ${ }^{3}$ \\ Jamile Guarda ${ }^{4}$ \\ Guilherme Liziero Ruggio da Silva ${ }^{5}$ \\ Antônio Cezar Faria Vilela ${ }^{6}$ \\ Eduardo Osório 6
}

\begin{abstract}
Resumo
$\mathrm{Na}$ literatura são descritos basicamente dois modelos de relações entre resistência mecânica e parâmetros microestruturais: (1) relações entre parâmetros microestruturais médios do coque e índices de ensaios mecânicos e (2) quantificação dos poros mais críticos a resistência mecânica, a partir de determinados critérios que levam em conta o tamanho e a forma dos poros. Nesse trabalho coques laboratoriais produzidos a partir de carvões com propriedades distintas foram caracterizados pela associação das técnicas de microscopia ótica e análise de imagens. Os principais modelos da literatura de relação entre resistência mecânica e parâmetros microestruturais foram avaliados. Foi demonstrado que nenhuma das relações estudadas conseguem descrever o comportamento mecânico de coques produzidos a partir de carvões de alto teor de componentes inertes $(>40 \%)$. A partir desses resultados duas novas abordagens foram realizadas a fim de obter modelos que contemplem tais coques: (1) introdução de uma nova relação entre resistência mecânica e os parâmetros microestruturais e (2) um novo critério de classificação das regiões derivadas de componentes inertes como críticas a resistência mecânica.
\end{abstract}

Palavras-chave: Coque metalúrgico; Microestrutura porosa de coque; resistência mecânica

\section{COKE MICROSTRUCTURE: RELATIONSHIP WITH MECHANICAL STRENGTH Abstract}

In the literature, two models of relationships between mechanical strength and microstructural parameters are described: (1) relations between average microstructural parameters and mechanical test indices; and (2) quantification of the most critical pores for mechanical strength, based on certain criteria take into account the size and shape of the pores. In this work, laboratory cokes waere produced by parent coals with large variety of properties. This cokes were characterized by the association of optical microscopy and image analysis techniques. The main models of the literature on the relationship between mechanical strength and microstructural parameters were evaluated. It has been demonstrated that none of the studied relationships can describe the mechanical behavior of cokes produced from high inert components (> 40\%). From these results two new approaches have been carried out to obtain models that contemplate such cokes: (1) introduction of a new relationship between mechanical strength and microstructural parameters and (2) a new criteria for classification of regions derived from inert components such as critical to mechanical strength.

Keywords: Coke; Coke Microstructure; Mechanical Strength

\footnotetext{
Mestre, Laboratório de Siderurgia (LaSid) da UFRGS, Porto Alegre, RS, Brasil.

Engenheiro, Laboratório de Siderurgia (LaSid) da UFRGS, Porto Alegre, RS, Brasil.

Doutor, Laboratório de Siderurgia (LaSid) da UFRGS, Porto Alegre, RS, Brasil.

Graduanda em engenharia, Laboratório de Siderurgia (LaSid) da UFRGS, Porto Alegre, RS, Brasil.

Doutor, Gerdau Açominas, Ouro Branco, MG, Brasil.

Professor Dr., Laboratório de Siderurgia (LaSid) da UFRGS, Porto Alegre, RS, Brasil.
} 


\section{INTRODUÇÃO}

A adequada marcha do alto-forno está intimamente relacionada com as propriedades do coque metalúrgico, que é produzido a partir da carbonização de misturas de carvões. Entre essas propriedades fundamentais do coque está a resistência mecânica, que é fortemente influenciada pela sua microestrutura porosa. O termo microestrutura porosa ou simplesmente microestrutura de coque refere-se à distribuição espacial da matriz carbonosa e sua porosidade. A caracterização completa da microestrutura porosa de coque é obtida pela associação das técnicas de microscopia ótica e análise de imagem [1-2].

A grande dificuldade atrelada a caracterização da microestrutura porosa do coque consiste na heterogeneidade de parâmetros físicos, como por exemplo a porosidade e densidade aparente, que podem variar significativamente nas diferentes regiões material. A essa heterogeneidade física é atribuído dois fatores: (1) o elevado gradiente térmico existente durante o processo de coqueificação, culminando na passagem pelo estágio plástico das diferentes regiões do material carbonizado em momentos distintos [3,4]; e (2) pelo comportamento dos componentes macerais dos carvões durante a carbonização. Para carvões coqueificáveis, os componentes macerais do grupo da vitrinita, liptinita, bem como um terço do maceral semifusinita [5], são chamados de macerais reativos, sendo os responsáveis pela formação da estrutura porosa do coque [6] denominada de regiões derivadas de componentes reativos (RDCR). Os demais macerais do grupo da inertinita, bem como aditivos alternativos (coque de petróleo, carvão não coqueificáveis, carvão vegetal, etc.) são chamados de componentes inertes, dando origem as regiões derivadas de componentes inertes (RDCI), que são caracterizadas por áreas densas de contornos retos, típicas de materiais não fundidos.

Diversas relações foram desenvolvidas entre parâmetros microestruturais e índices de ensaios de resistência mecânica, no entanto identifica-se que essas relações são muito influenciadas por as particularidades de cada métodos de caracterização microestrutural. Por essa razão a aplicabilidade dessas relações é fortemente limitada. Além disso, apesar de existirem estudos que mostraram isoladamente a influência de parâmetros microestruturais das RDCR [7-13] e RDCl [14-20] na resistência mecânica do coque, não existe uma relação eficaz que unifique a contribuição dessas duas regiões.

A partir do exposto, esse trabalho tem como objetivo principal caracterizar a microestrutura dos coques, através de parâmetros que descrevam as áreas derivadas de componentes reativos e inertes, avaliar relações da literatura entre parâmetros microestruturais e a resistência mecânica do coque e por fim desenvolver novas relações que unifiquem parâmetros microestruturais das RDCR e $\mathrm{RDCl}$ dos coques, visando a descrição completa do seu comportamento mecânico.

\section{MATERIAIS E MÉTODOS}

\section{Coques de estudo}

Os coques estudados foram produzidos em laboratório a partir de carvões (A1, A2, M1 e M2) e misturas (MC1 e MC2) de carvões tipicamente utilizados industrialmente em siderúrgicas para produção de coque (Tabela 1). Segundo a norma ASTM D388, os carvões do estudo podem ser classificados como: A1 e A2 carvões betuminosos de alta matéria volátil, M1 e M2 carvões betuminosos de média matéria volátil e MC1 e MC2 misturas de carvões com médio teor de matéria volátil. 
Tabela 1. Análise imediata dos carvões individuais e misturas de estudo.

\begin{tabular}{|c|c|c|c|c|c|c|c|c|c|c|}
\hline Carvões & $\underset{\text { bsic (\%) }}{\text { MV }}$ & $\begin{array}{c}\mathbf{C z}_{(\%)} \text { bs } \\
\text { (1) }\end{array}$ & $\underset{(\%)}{\mathbf{C}_{\mathrm{F} \text { bs }}}$ & $\underset{(\%)}{\mathbf{R m}}$ & $\underset{\text { imm }}{\mathbf{V}_{\text {Vol.\% }}}$ & $\underset{\text { imm }}{L_{\text {Vol.\% }}}$ & $\begin{array}{c}I^{*} \text { Vol.\% } \\
\text { imm }\end{array}$ & $\begin{array}{l}\text { MM } \\
\text { Vol. } \%\end{array}$ & $\begin{array}{l}\text { IP } \\
\left({ }^{\circ} \mathrm{C}\right)\end{array}$ & $\begin{array}{c}\text { MF } \\
\text { (ddpm) }\end{array}$ \\
\hline A1 & 38,8 & 7,5 & 56,6 & 0,912 & 83,0 & 3,0 & 14,0 & 7,2 & 103 & 46334 \\
\hline A2 & 36,8 & 6,8 & 58,8 & 0,841 & 78,0 & 10,0 & 11,9 & 4,4 & 84 & 11952 \\
\hline M1 & 22,6 & 9,6 & 70,0 & 1,159 & 64,4 & 0,0 & 35,6 & 10,0 & 44 & 3 \\
\hline M2 & 22,8 & 9,5 & 70,0 & 1,207 & 81,3 & 0,4 & 18,3 & 7,2 & 77 & 320 \\
\hline MC1 & 27,6 & 8,7 & 66,0 & 1,018 & 78,8 & 0,9 & 20,3 & 7,6 & 78 & 274 \\
\hline MC2 & 22,5 & 6,5 & 72,5 & 1,119 & 60,1 & 3,4 & 36,5 & 10,8 & 78 & 327 \\
\hline
\end{tabular}

$\overline{\mathrm{MV}}=$ Matérial volátil; CZ = Cinzas; CF = Carbono Fixo, bs = base seca; bsic = base seca isenta de cinzas; $\mathrm{Rm}=$ refletância média da vitrinita; $V=$ Vitrinita; $L=$ Liptinita; $I^{*}=$ Macerais do grupo da Inertinita + Coque de petróleo; $\mathrm{MM}=$ Matéria Mineral; \%Vol. = Percentual volumétrico; imm = isento de matéria mineral; IP = intervalo plástico e $\mathrm{MF}=$ máxima fluidez.

\section{Caracterização física dos coques}

A Tabela 2 apresenta a caracterização física dos coques estudados nos ensaios de tamboramento (l600) e compressão diametral (S). Além do valor da média nos ensaios, um parâmetro referente a variabilidade (erro padrão) também encontra-se na tabela.

Tabela 2. Caracterização da resistência mecânica dos coques estudados.

\begin{tabular}{|c|c|c|c|c|c|c|}
\hline & $\mathrm{C}_{\mathrm{A} 1}$ & $\mathrm{C}_{\mathrm{A2}}$ & $\mathrm{C}_{\mathrm{M} 1}$ & $\mathrm{C}_{\mathrm{M} 2}$ & $\mathrm{C}_{\mathrm{MC1}}$ & $\mathrm{C}_{\mathrm{MC2}}$ \\
\hline$\overline{S_{(\mathrm{MPa})}}$ & 6,3 & 6,0 & 8,6 & 9,8 & 8,2 & 8,2 \\
\hline Erro padrão - S (MPa) & 0,5 & 0,6 & 0,5 & 0,4 & 0,6 & 0,6 \\
\hline$I_{600}(\%)$ & 74,1 & 77,3 & 77,7 & 84,5 & 83,2 & 83,2 \\
\hline Erro padrão - $I_{600}(\%)$ & 0,2 & 0,1 & 0,3 & 0,0 & 0,1 & 0,4 \\
\hline
\end{tabular}

\section{Caracterização da microestrutura dos coques}

A caracterização da microestrutura do coque foi realizada pela associação das técnicas de microscopia ótica e análise de imagem, seguindo a metodologia descrita em Agra, et al. (2018) [1-2]. Cinco plugs de coque foram produzidos a partir de partículas com granulometria entre 19 e 22,5 $\mathrm{mm}^{2}$. Para cada um dos plugs produzidos foram capturadas imagens com dimensões de $56,7 \mathrm{~mm}^{2}$ $(0,544$ pixels $/ \mu \mathrm{m})$ em microscópio ótico petrográfico Leica DM6000 M (lente objetiva de $5 x$ ). A análise dessas imagens foi efetuada através do desenvolvimento de uma macro no software de domínio público ImageJ (National Institutes of Health). Essa macro, que consiste em uma sequência de operações, fornece informações referente as regiões derivadas dos componentes reativos (RDCR) e inertes (RCDI) do coque. O fluxograma dessa rotina de análise é apresentado na Figura 1.

Uma imagem típica de microestrutura porosa de coque obtida em microscópio ótico é apresentada na Figura 2a. Essa imagem em tons de cinza é utilizada como input na análise. Inicialmente são segmentados as áreas referentes a matriz carbonosa e os poros do coque. Essa segmentação é realizada com auxílio da ferramenta threshold e resultando em uma imagem binária (Figura $2 \mathrm{~b}$ ), onde as áreas em branco representam a matriz carbonosa e preto os poros. Em seguida as RDCI são distinguidas das demais regiões por morfologia e pintadas de branco. Essa etapa resulta em duas novas imagens: a Figura 2c, em que as RDCI (pintadas de branco) estão presentes juntamente com as RDCR e a Figura $2 \mathrm{~d}$, onde apenas as RDCl (pintadas de branco) estão presentes. Essas duas imagens foram utilizadas para obter os parâmetros microestruturais das RDCR e RDCI, respectivamente (conforme o fluxograma da Figura 1). 


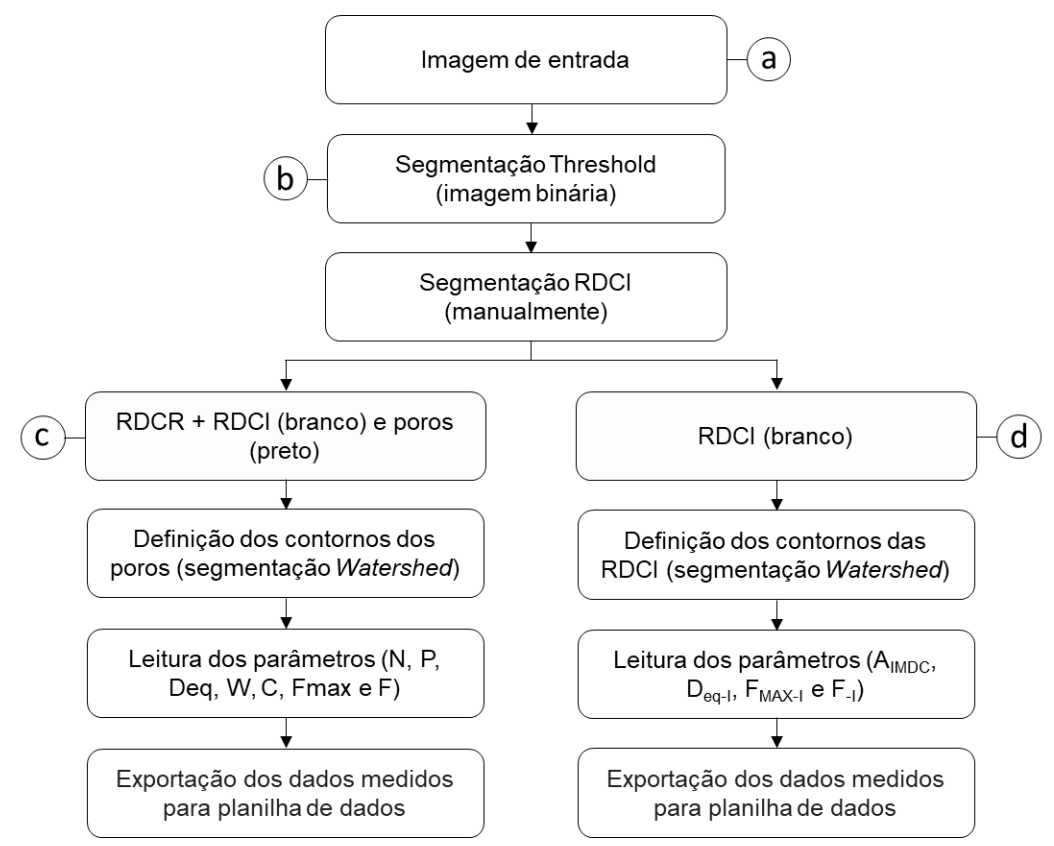

Figura 1. Fluxograma de análise das imagens de coque
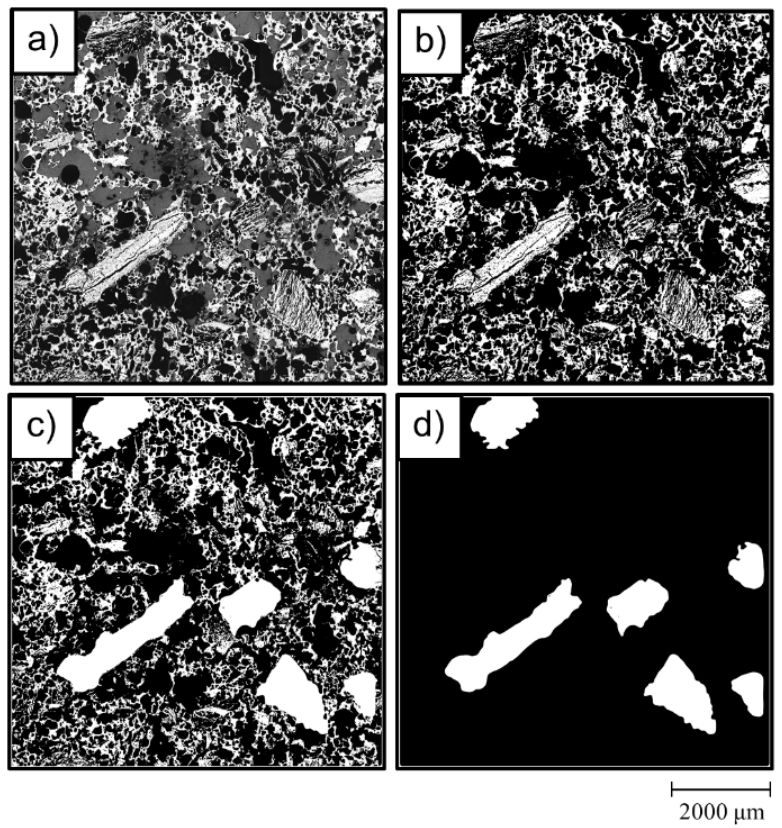

Figura 2. Passos de operações para obtenção dos parâmetros da microestrutura porosa do coque.

Os parâmetros obtidos na análise referentes as RDCR e RDCI são apresentados na Figura 3, juntamente com a representação e a descrição básica. Parâmetros tais como porosidade, número de poros por área, tamanho médio de poro e espessura média de parede descrevem a amostra como um todo (parâmetros globais). No entanto a análise dos poros de forma individual (tamanho e forma) torna-se interessante com vistas a quantificar os poros mais críticos para a resistência mecânica do coque. Essa quantificação dos poros críticos foi feita realizada com base em trabalhos que apontam que poros com diâmetro equivalente superior a 300 $\mu \mathrm{m}$ e/ou com circularidade inferior a 0,2 são os mais nocivos a resistência mecânica do coque [15-17]. De forma análoga as RDCI também podem ser classificadas como críticas (ou não) para a resistência mecânica. Para as $\mathrm{RDCl}$ adotou-se os seguintes 
critérios para classificação como críticas: RDCl com $F_{\max }$ superior a 1,5 mm [18] e/ou perímetro > $1000 \mu \mathrm{m}$.

\begin{tabular}{|c|c|c|}
\hline Parâmetros & Representação & Descrição básica \\
\hline Porosidade (\%) & & $\begin{array}{l}\text { Contagem da área ocupada por pixels pretos em relação a área total } \\
\text { considerada. }\end{array}$ \\
\hline Número de poros por $\mathrm{mm}^{2}$ & & Quantidade de poros medidos por uma determinada área da imagem. \\
\hline Diâmetro Equivalente $(\mu \mathrm{m})$ & & $\begin{array}{l}\text { Diâmetro de um círculo perfeito que equivale a área do poro. Poros com Deq > } \\
300 \mu m \text { são classificados como críticos a resistência mecânica. }\end{array}$ \\
\hline Circularidade $\quad C=\frac{4 \pi \times A}{P^{2}}$ & & $\begin{array}{l}\text { Fator atrelado a forma do poro, que faz relação entre área e perímetro. } \\
\text { Circularidade }=1 \text { para círculos perfeitos. Poros } \operatorname{com} C<0,2 \text { são classificados } \\
\text { como críticos a resistência mecânica. }\end{array}$ \\
\hline Espessura de parede $(\mu \mathrm{m})$ & & $\begin{array}{l}\text { Comprimento médio de retas que cruzam as paredes dos coques em dada } \\
\text { direção. }\end{array}$ \\
\hline $\begin{array}{l}\text { Diâmetro máximo de Feret } \\
(\mu \mathrm{m})\end{array}$ & & $\begin{array}{l}\text { A distância entre duas retas mais distantes que tangenciam o contorno de um } \\
\text { objeto. }\end{array}$ \\
\hline Relação Feret $=\frac{\text { Fmax }}{\text { Fmin }}$ & & $\begin{array}{l}\text { A razão entre as retas mais distantes e mais próximas que tangenciam o } \\
\text { contorno de um objeto. }\end{array}$ \\
\hline Diâmetro Equivalente $(\mu \mathrm{m})$ & & Diâmetro de um círculo perfeito que equivale a área do inerte. \\
\hline Área RDCl (\%) & & $\begin{array}{l}\text { Percentual de área ocupada pelas RDCI na área total da matriz carbonosa do } \\
\text { coque. }\end{array}$ \\
\hline $\begin{array}{l}\text { Diâmetro máximo de Feret } \\
(\mu \mathrm{m})\end{array}$ & & $\begin{array}{l}\text { A distância entre duas retas mais distantes que tangenciam o contorno de um } \\
\text { objeto. Quando o Fmax }>1,5 \mathrm{~mm} \text {, esta RDCl é classificado como crítico. }\end{array}$ \\
\hline Perímetro $(\mu \mathrm{m})$ & & $\begin{array}{l}\text { Medida do contorno de uma RDCl. Quando o perímetro }>1000 \mu \mathrm{m} \text {, esta RDCl é } \\
\text { classificada como crítica. }\end{array}$ \\
\hline
\end{tabular}

Figura 3. Parâmetros avaliados da microestrutura porosa de coque.

\section{Relação da microestrutura dos coques com a resistência mecânica}

As principais modelos de descrição da resistência mecânica do coque através dos parâmetros microestruturais foram avaliados nesse estudo, com vistas a identificar qual abordagem melhor se adapta aos dados obtidos nesse trabalho.

Dois grupos de modelos foram testados: (1) modelos que relacionam a resistência mecânica com parâmetros microestruturais globais do coque (porosidade, tamanho de poro médio, etc.) e (2) modelos que relacionam a resistência mecânica do coque com os poros mais críticos a resistência mecânica (poros de determinada forma e tamanho). Em seguida, novos modelos derivados dos anteriores foram propostos visando a inclusão de parâmetros referentes as $\mathrm{RDCl}$ dos coques.

\section{RESULTADOS E DISCUSÃO}

\subsection{Caracterização da microestrutura dos coques}

Os parâmetros microestruturais dos coques estudados, obtidos pela metodologia anteriormente descrita, são apresentados na Tabela 3.

De maneira geral pode-se observar que os coques produzidos a partir dos carvões de alta matéria volátil (A1 e A2) deram origem aos coques de alta porosidade $(66,4$ e $64,4 \%)$, com poros de grandes dimensões (257 e $259 \mu \mathrm{m})$ e com as relações entre a espessura média das paredes e o tamanho médio dos poros (W/Deq) mais baixas entre os materiais analisados $(0,36$ e 0,43$)$. 
Tabela 3. Parâmetros microestruturais dos coques laboratoriais estudados.

\begin{tabular}{|c|c|c|c|c|c|c|c|c|c|c|c|c|}
\hline & \multicolumn{7}{|c|}{ RDCR } & \multicolumn{5}{|c|}{ RDCl } \\
\hline & $\begin{array}{c}\mathbf{N} \\
\left(\text { poros } / \mathrm{mm}^{2}\right)\end{array}$ & $\begin{array}{l}\mathbf{P} \\
(\%)\end{array}$ & $\begin{array}{l}\mathbf{D}_{\mathrm{eq}} \\
(\mu \mathrm{m})\end{array}$ & $\underset{(\mu \mathrm{m})}{\mathbf{W}}$ & $\begin{array}{l}\mathbf{C} \\
(-)\end{array}$ & $\begin{array}{c}\mathbf{F}_{\text {MAX }} \\
(\mu \mathrm{m})\end{array}$ & $\begin{array}{l}\mathbf{F} \\
(-)\end{array}$ & $\begin{array}{c}\mathbf{A}_{\mathrm{RDCl}} \\
(\%)\end{array}$ & $\begin{array}{l}\mathrm{D}_{\text {eq-1 }} \\
(\mathrm{mm})\end{array}$ & $\underset{(\mathrm{mm})}{\mathbf{P}}$ & $\underbrace{}_{(\mu \mathrm{m})} \mathrm{F}_{\text {MAX-II }}$ & $\begin{array}{l}\mathbf{F}_{-\mid} \\
(-)\end{array}$ \\
\hline$\overline{C_{A 1}}$ & 11,9 & 66,4 & 257 & 92 & 0,53 & 313 & 1,58 & 2,0 & 0,53 & 2,7 & 930 & 2,8 \\
\hline $\mathrm{C}_{\mathrm{A} 2}$ & 12,0 & 64,4 & 259 & 111 & 0,63 & 300 & 1,54 & 12,6 & 0,93 & 14,9 & 1426 & 3,2 \\
\hline $\mathrm{C}_{\mathrm{M} 1}$ & 15,5 & 44,5 & 189 & 110 & 0,40 & 256 & 1,62 & 36,5 & 1,99 & 9,5 & 3244 & 2,4 \\
\hline $\mathrm{C}_{\mathrm{M} 2}$ & 15,1 & 51,0 & 219 & 109 & 0,49 & 281 & 1,61 & 8,00 & 1,04 & 5,0 & 1541 & 2,3 \\
\hline $\mathbf{C}_{\mathrm{MC} 1}$ & 15,8 & 57,6 & 212 & 90 & 0,41 & 275 & 1,60 & 17,6 & 1,14 & 4,9 & 1562 & 2,0 \\
\hline $\mathrm{C}_{\mathrm{MC} 2}$ & 13,0 & 47,4 & 213 & 111 & 0,32 & 284 & 1,63 & 21,0 & 1,27 & 5,9 & 1721 & 1,6 \\
\hline
\end{tabular}

Os demais coques, produzidos pelos carvões individuais (M1 e M2) e misturas (MC1 e MC2) de média matéria volátil, apresentaram porosidade e tamanho de poros inferiores aos coques $C_{A 1}$ e $C_{A 2}$. No entanto, é interessante fazer a distinção entre os coques $\mathrm{C}_{\mathrm{M} 1}$ e $\mathrm{C}_{\mathrm{MC} 2}$, dos carvões de alto teor de componentes inertes (vide Tabela 1) e os coques $\mathrm{C}_{M 2}$ e $\mathrm{C}_{M C 1}$. $\mathrm{O}$ alto teor de inertes na composição maceral do carvão $\mathrm{M} 1$ e da mistura de carvões MC2 é o fator responsável pela grande fração de área da matriz carbonosa correspondente as $\mathrm{RDCl}\left(\mathrm{ARDCl}_{\mathrm{R}}\right)$ ), com os maiores valores entre os coques analisados (36,5 e 21,0\%). Além disso, esses coques também apresentam as $\mathrm{RDCl}$ de maiores dimensões.

Os coques $\mathrm{C}_{\mathrm{M} 2}$ e $\mathrm{C}_{\mathrm{MC} 1}$ apresentaram as microestruturas mais regulares entre os coques analisados, com porosidade e tamanho de poros em níveis intermediários aos coques produzidos a partir dos carvões de alta matéria volátil (A1 e A2) e ricos em inertes (M1 e MC2).

Outro ponto importante interessante a ser analisado é a forma dos poros dos coques analisados, que é descrita pela circularidade (c) e relação Feret (F). Como mostra a Tabela 3 a forma dos poros tende a ser mais acircular quanto maior a fração de área de RDCI na sua microestrutura.

\subsection{Relação de múltiplas variáveis de parâmetros microestruturais}

\section{Tensão de compressão diametral e parâmetros microestruturais}

As principais relações descritas na literatura entre os parâmetros microestruturais e a tensão de compressão diametral do coque são apresentadas na Tabela 4. A partir dessas relações, uma série de equações foram realizadas e apresentadas juntamente na tabela. Os coeficientes de determinação das relações servem como quantificadores do poder de descrição da resistência mecânica pelos parâmetros microestruturais nas equações testadas.

As relações iniciais ( $R 1$ a R5) são tipicamente utilizadas na descrição do comportamento mecânico de materiais porosos em geral. Nessas relações é admitido que a porosidade sozinha é suficiente para explicar a capacidade do material de suportar determinada tensão antes da ruptura. A relação R6, por sua vez, é uma adaptação da R5, adicionando a essa outros parâmetros microestruturais, o diâmetro máximo de Feret $\left(F_{\max }\right)$ que está relacionado com o tamanho dos poros e a relação Feret (F) que está relacionado com a forma dos poros. Por fim, as relações R7 a R10 utilizam múltiplos parâmetros microestruturais na tentativa da descrição mecânica. 
Tabela 4. Relações da resistência a compressão (S) com parâmetros microestruturais dos coques laboratoriais de estudo.

\begin{tabular}{|c|c|c|c|c|}
\hline \multirow{2}{*}{$\begin{array}{c}\text { Referência } \\
-\end{array}$} & \multirow{2}{*}{$\begin{array}{l}\text { Relação } \\
S \alpha(1-p)\end{array}$} & \multicolumn{2}{|c|}{ Equação adaptada } & \multirow{2}{*}{$\frac{\mathbf{R}^{2}}{0,66}$} \\
\hline & & $\mathbf{R 1}$ & $\mathrm{S}=12,84 \mathbf{R} 1+2,11$ & \\
\hline [8] & $S \alpha 1-1,21 p^{2 / 3}$ & $\mathbf{R 2}$ & $S=21,79 \mathbf{R} 2+4,09$ & 0,62 \\
\hline [9] & $S \alpha 1-1,21 p^{2 / 3} /\left(1+0,437 p+2,41 p^{5 / 3}\right)$ & R3 & $S=12,95 \mathbf{R 3}+5,43$ & 0,65 \\
\hline [10] & $S \alpha(1-p) /(1+3 p)$ & R4 & $S=20,64 \mathbf{R 4}+5,94$ & 0,60 \\
\hline [11] & $S \alpha \exp (-b p)$ & R5 & $S=22,04 \mathbf{R 5}-4,87$ & 0,64 \\
\hline [12] & $\left.S \alpha \mathrm{Fmax}^{-1 / 2} \exp \left(-2(\mathrm{~F})^{0,5} \mathrm{p}\right)\right)$ & R6 & $S=301,81 \mathbf{R} 6+3,31$ & 0,59 \\
\hline$[12]$ & S.N $\alpha W / D_{\text {eq }}$ & R7 & $S . N=247,76 \mathbf{R 7}-5,37$ & 0,41 \\
\hline [13] & S.N $\propto 10^{3} \mathrm{~W} / \mathrm{D}_{\mathrm{eq}}{ }^{2}$ & $\mathbf{R 8}$ & S.N = 36,91 R8 + 31,7 & 0,51 \\
\hline [14] & $S \propto W / D_{\text {eq }}$ & $\mathbf{R 9}$ & $S=11,96 \mathbf{R} \mathbf{9}+2,25$ & 0,47 \\
\hline [15] & $S \propto W . S_{f} \cdot R_{f} / p . D_{e q}$ & $\mathbf{R} 10$ & $\mathrm{~S}=4,00 \mathbf{R} \mathbf{1 0}+4,84$ & 0,33 \\
\hline
\end{tabular}

As equações produzidas a partir das relações que levam em conta apenas a porosidade (R1 a R5) apresentaram as correlações mais fortes com o índice de resistência a compressão diametral. Esse resultado não é compatível com o observado nos experimentos de Patrick (1983) [12], onde foi demonstrado que a aplicação da relação R5 para uma série de coques, incluindo amostras de escalas laboratoriais, piloto e industrial apresentou correlação limitada $(R=0,48)$. Para os coques do presente estudo entende-se que a descrição da resistência mecânica apenas pela porosidade apresenta bom nível de correlação devido ao nível de porosidade bastante distinto entre os materiais analisados. Para o caso de aplicação industrial onde os coques utilizados apresentam um intervalo de porosidade bem restrito a descrição da resistência mecânica a partir da não é suficiente. Brown et al. (1964) [20], mostrou que a descrição do comportamento mecânica por meio de parâmetros microestruturais, para materiais com porosidade do nível coque metalúrgico, deve levar em conta outros parâmetros microestruturais, como o tamanho e a formas dos poros, como as relações $\mathrm{R} 6$ a $\mathrm{R} 10$.

Entre essas relações a R6 e R8 apresentaram as melhores adaptações para os dados desse trabalho, com coeficientes de determinação de 0,59 e 0,51, respectivamente. Apesar disso esse nível de correlação não é suficientemente elevado para a caracterização de uma correlação muito forte, ou em outras palavras, que os parâmetros microestruturais contidos nelas são suficientes para descrever o comportamento mecânico do material.

Conforme foi descrito anteriormente, a microestrutura do coque é composta pela matriz carbonosa e pelos poros. A matriz carbonosa por sua vez é composta por duas regiões: RDCR e inertes RDCl. Para a descrição da RDCR (matriz e poros), os parâmetros relevantes são: porosidade, tamanho de poros, forma dos poros e espessura de parede. Todos esses parâmetros são contemplados em pelo menos uma das relações $\mathrm{R} 6$ e R8. No entanto, nenhuma das relações da Tabela 4 traz qualquer parâmetro que descrevam as $\mathrm{RDCl}$ dos coques.

Conforme pode ser visto na Tabela 1, a composição maceral do carvão M1 e da mistura MC2 contém 45,6 e 47,3\% de componentes inertes (macerais do grupo da inertinita + aditivos inertes + matéria mineral) em volume, respectivamente. Essa grande quantidade de componentes inertes resulta nas elevadas áreas de RDCl observadas na Tabela 3. A partir disso é natural supor que a descrição do comportamento mecânico desses coques apenas por parâmetros referentes às RDCR não é insuficiente, pois os elevados volumes das RDCl presentes nos coques 
não podem ser negligenciados. Nesse trabalho os coques produzidos por esses tipos de carvões serão chamados de coques saturados em inerte.

\section{Índice de tamboramento e parâmetros microestruturais}

O ensaio de resistência mecânica por compressão diametral caracteriza-se por ser um ensaio mecânico estático, onde basicamente as solicitações mecânicas existentes consistem em tensões de compressão no sentido de aplicação de força e tensões trativas no plano perpendicular. Por outro lado, o ensaio de tamboramento é um ensaio dinâmico, onde as solicitações mecânicas presentes são abrasão e impacto. Apesar dessas significativas diferenças entre avaliou-se a adaptação das equações derivadas das relações R6 e R8 para descrever também o índice de tamboramento (l600) pelos parâmetros microestruturais (R6 e R8). Essa adaptação também é motivada pelo fato que os ensaios de tamboramento são os ensaios mais utilizados atualmente nas usinas siderúrgicas para avaliar a degradação mecânica de coque, tanto a frio quanto a quente.

As equações que melhor se adaptaram aos dados desse estudo, realizadas a partir das relações $\mathrm{R} 6$ e $\mathrm{R} 8$ tiveram coeficiente de determinação com valores baixos 0,20 e 0,14 , respectivamente. Análogo ao ensaio de compressão diametral, entende-se que essas baixas correlações observadas se devem ao fato de não existir nenhum parâmetro microestrutural referente as $\mathrm{RDCl}$ nas relações utilizadas (R6 e R8). A partir disso sugere-se a incorporação de novos parâmetros nessas relações.

\section{Modificação das relações pela inclusão de parâmetros das RDCI}

Conforme comentado anteriormente nenhuma das relações anteriormente avaliadas tiveram resultados satisfatórios com os dados desse trabalho. A partir da análise criteriosa dos materiais foi observado que dois coques em especial possuem um volume de $\mathrm{RDCl}$ extremamente elevado. $\mathrm{Na}$ literatura é descrito que a resistência mecânica é fortemente influenciada pelas $\mathrm{RDCl}$, principalmente pelos seguintes mecanismos: problemas de interfaces entre as RDCR e RDCl [21-22], distorções e tensionamento da matriz causada pelo volume do inerte [18]. A partir desses levantamentos da literatura e dos parâmetros da Tabela 3, sugere-se a introdução de uma nova relação envolvendo parâmetros microestruturais exclusivos das RDCl $\left(R 11 \rightarrow S\right.$ a $10^{-5}$.ARDCI.Deq-I). Basicamente essa relação indica que a resistência mecânica do coque é influenciada tanto pela quantidade das $\mathrm{RDCl}$, mas também pelas dimensões das mesmas. Obviamente por tratar-se de uma influencia de deterioração um sinal negativo deve ser observado nas equações derivadas de tal relação. Ademais, o fator multiplicador $\left(10^{-5}\right)$ é simplesmente uma constante de proporcionalidade.

A Equação 1 é sugerida como a forma genérica da descrição de um índice de ensaio mecânico (RM) por parâmetros microestruturais das RDCR (R8) e RDCl (R11). Onde, a, b e c são ponderadores dos efeitos relativos, diferentes para cada tipo de solicitação mecânica.

$\mathrm{RM}=\mathrm{a} \cdot \mathbf{R} \mathbf{8}+\mathrm{b} \cdot \mathbf{R} \mathbf{1 1}+\mathrm{c}$

A partir das relações utilizadas ( $R 8$ e R11) e dos valores dos índices dos ensaios mecânicos (Tabela 2), calculou-se por regressão as equações 2 e 3 que descrevem o comportamento mecânico dos ensaios de tamboramento e compressão diametral a partir dos parâmetros microestruturais, respectivamente. A Figura 4 mostra as relações entre os índices medidos (Tabela 2) e calculados (equações 2 e 3). 
$\mathrm{I}_{600}=12,58 \cdot \mathbf{R} \mathbf{8}-26,80 \cdot \mathbf{R} 11+59,28$

$\mathrm{S}=4,32 \cdot \mathbf{R} 8-7,03 \cdot \mathbf{R} \mathbf{1 1}+0,25$
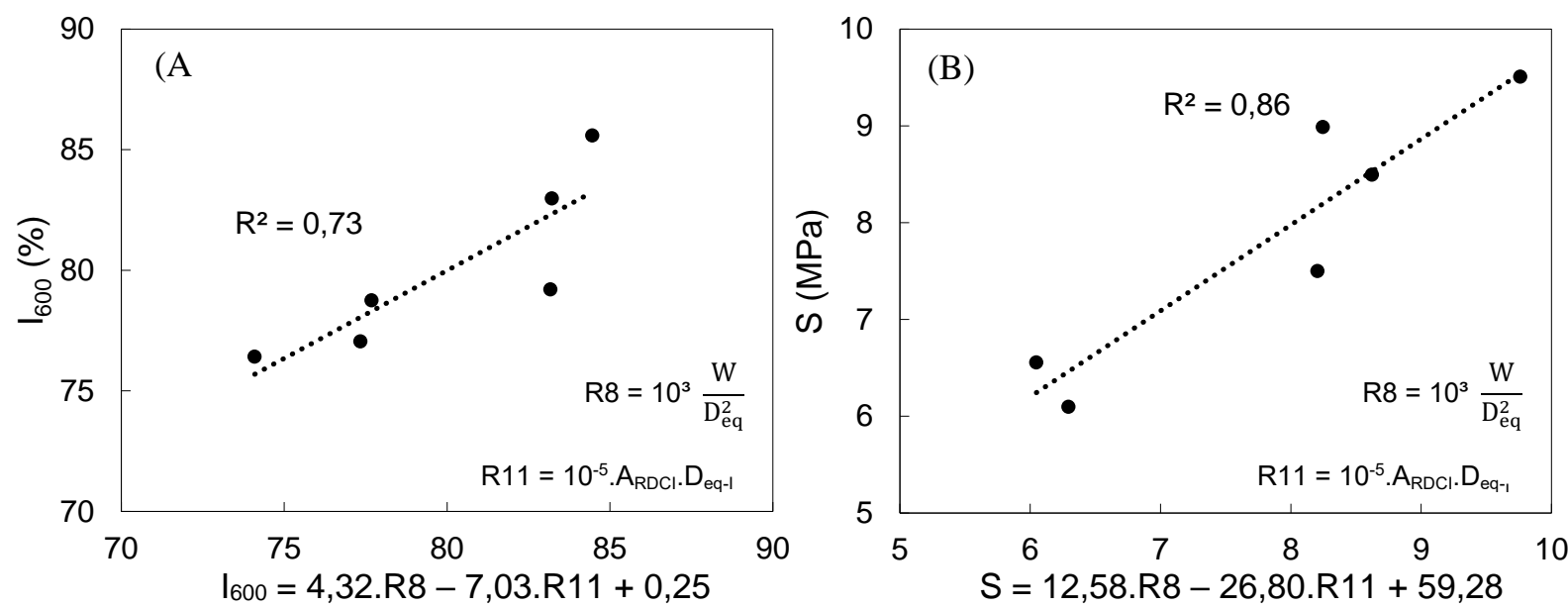

Onde, $\mathbf{R} 8=10^{3} \cdot \frac{\mathrm{W}}{\mathrm{D}_{\mathrm{eq}}{ }^{2}}$ e $\mathbf{R} \mathbf{1 1}=10^{-5} \cdot$ ARDCI. $_{\text {eq-I }}$.

Figura 4. Relação entre os índices medidos e estimados nos testes de (a) tamboramento e (b) compressão diametral.

Conforme pode ser observado na Figura $4 a-b$ as correspondências dessas equações, para ambos ensaios, aumentaram notavelmente com a incorporação do fator relativo às $\mathrm{RDCl}$ (R11). A título de comparação, o coeficiente de determinação entre o índice de tamboramento, apenas com a relação dos parâmetros das RDCR (R8), passou de 0,14 para 0,73 e o coeficiente de determinação para o ensaio de compressão diametral passou de 0,51 para 0,86.

Além do aumento da acurácia das relações, outras informações podem ser extraídas dessas equações é o sinal dos índices assumidos para as diferentes relações. Como pode ser visto nas equações 2 e 3 os sinais indicam a relação positiva entre a resistência mecânica e a R8, porem negativa para a R11. Em outras palavras isso quer dizer que quanto maior a relação R8, mais favorecida é a resistência mecânica. Ou seja, quanto maior a espessura das paredes e menor o tamanho dos poros melhor o comportamento mecânico do coque, concordando com trabalhos anteriores $[12,14]$. Quanto ao sinal negativo atribuído a relação $R 11$, indica que quanto maior a área da matriz carbonosa compostas por $\mathrm{RDCl}$ e maior forem essas regiões, maior o efeito depreciador dos inertes na resistência mecânica do coque, esse resultado também concorda com trabalhos anteriores [17-18].

\subsection{Relações entre resistência mecânica e as áreas críticas}

\section{Caracterização dos poros críticos a resistência mecânica}

Outra interpretação para descrever o comportamento mecânico do coque metalúrgico pode ser realizada através da quantificação dos poros mais susceptíveis a ruptura devido a uma tensão aplicada. Essa abordagem dá mais importância as características microestruturais com maior potencial de falha do que à média dos parâmetros. Nesse trabalho os poros críticos foram classificados conforme os 
critérios de tamanho e forma (Figura 3). A caracterização dos poros críticos dos coques de estudo é apresentada na Tabela 5.

Tabela 5. Poros críticos a resistência mecânica dos coques laboratoriais estudados.

\begin{tabular}{l|ccc|ccc}
\hline \multirow{2}{*}{ Coque } & \multicolumn{3}{|c|}{ Percentual numérico } & \multicolumn{3}{c}{ Percentual de área } \\
\cline { 2 - 7 } & $\mathbf{D}_{\mathrm{eq}} \mathbf{>} \mathbf{3 0 0} \boldsymbol{\mu m}$ & $\mathbf{C}<\mathbf{0 , 2}$ & TOTAL & $\mathbf{D}_{\mathrm{eq}}>\mathbf{3 0 0} \boldsymbol{\mu m}$ & $\mathbf{C}<\mathbf{0 , 2}$ & TOTAL \\
\hline $\mathbf{C}_{\mathrm{A} 1}$ & 19,3 & 2,8 & 21,1 & 59,8 & 5,3 & 60,7 \\
$\mathbf{C}_{\mathrm{A} 2}$ & 16,7 & 2,5 & 18,6 & 62,1 & 3,0 & 63,1 \\
$\mathbf{C}_{\mathrm{M} 1}$ & 6,5 & 9,4 & 14,7 & 28,5 & 13,4 & 36,2 \\
$\mathbf{C}_{\mathrm{M} 2}$ & 11,7 & 11,7 & 21,0 & 42,7 & 16,8 & 49,6 \\
$\mathbf{C}_{\mathrm{MC} 1}$ & 11,8 & 8,2 & 17,5 & 44,7 & 15,6 & 49,0 \\
$\mathbf{C}_{\mathrm{MC} 2}$ & 10,8 & 27,2 & 34,4 & 43,3 & 31,9 & 58,8 \\
\hline
\end{tabular}

As relações entre o volume dos poros críticos e os índices dos ensaios de resistência mecânica dos coques são apresentadas nas Figuras $5 \mathrm{a}$ e b. Como pode ser observado, os índices de determinação dessas relações são baixos, principalmente para a relação com o índice do ensaio de tamboramento. A partir da análise criteriosa das relações, dois pontos podem ser identificados como os mais discrepantes, que são justamente os coques $\mathrm{C}_{\mathrm{M} 1}$ e $\mathrm{C}_{\mathrm{MC} 2}$ saturados em inertes. Pela caracterização dos poros críticos o coque $\mathrm{C}_{\mathrm{M} 1}$ deveria apresentar resistência mecânica superior e o coque $\mathrm{C}_{\mathrm{MC}}$ inferior à obtida em ambos ensaios.
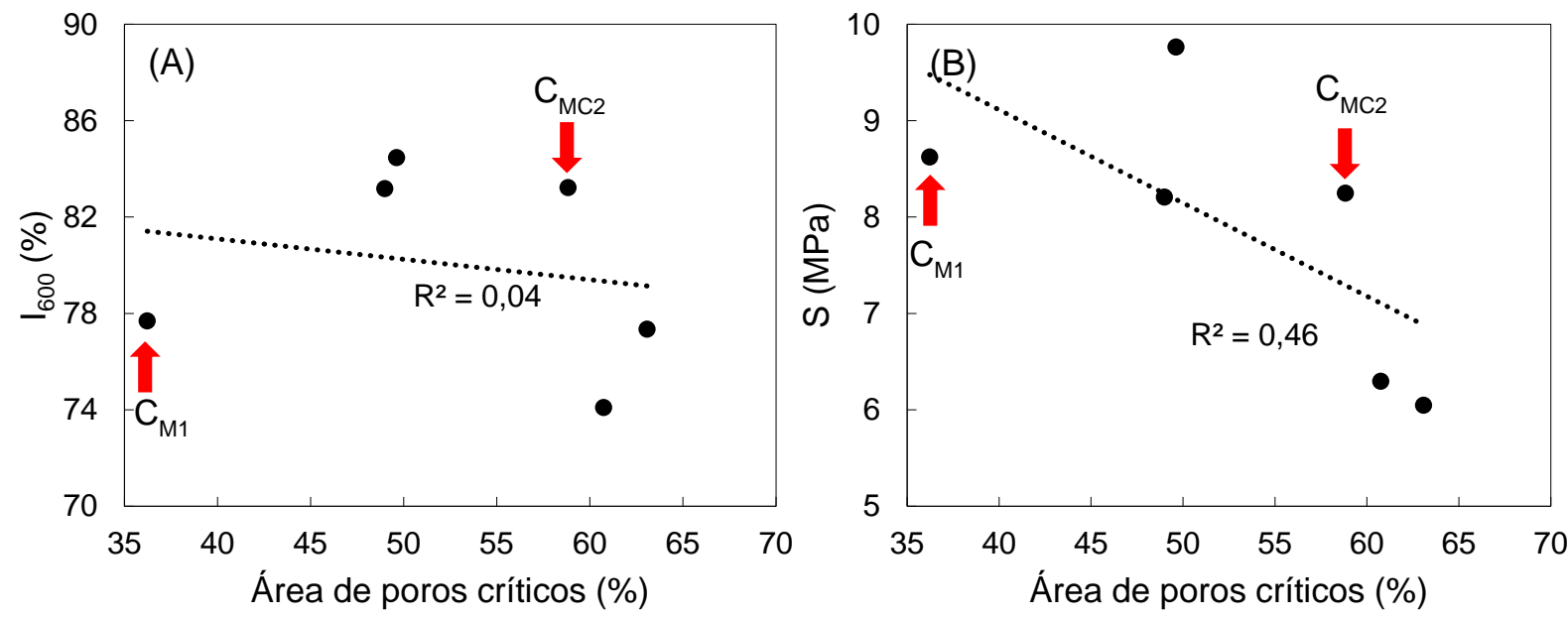

Figura 5. Relações dos poros críticos dos coques laboratoriais com (a) índice de tamboramento, (b) tensão de compressão diametral

Quando essas relações são refeitas, eliminando os coques saturados em inertes, identifica-se que o grau de correspondência das relações aumenta significativamente, passando de 0,04 para 0,83 no ensaio de tamboramento e de 0,46 para 0,84 no ensaio de compressão. A partir desses resultados, duas ações podem ser realizadas para melhor as relações com vistas a contemplar também os coques saturados em inertes: (1) Ponderamento diferenciado para os poros críticos pelo critério de tamanho e forma (2) Introdução de parâmetros relativos as regiões derivadas de componentes inertes.

\section{Inclusão de parâmetros de RDCI nas áreas críticas a resistência mecânica}

Com vistas a descrever os coques de alto teor de inertes, a abordagem da quantificação dos poros críticos pode ser ligeiramente modificada, porém mantendo a mesma lógica. Para contemplar os efeitos nocivos ocasionados pelos inertes na 
microestrutura dos coques é sugerido utilizar dois critérios de classificação de RDCI como mais críticas a resistência mecânica. Assim, a abordagem da quantificação de áreas de poros críticos, passa a ser, de modo mais generalista, quantificação das áreas críticas totais a resistência mecânica do coque, incluindo poros e RDCl.

A listagem dos parâmetros de classificações das áreas críticas do coque, provenientes dos poros (tamanho e forma), das $\mathrm{RDCl}$ (tamanho e perímetro) e totais são descritas a seguir:

1. $A$ Deq $>300=$ área de poros com $D_{\text {eq }}>300 \mu \mathrm{m}[15]$;

2. $A_{c<0,2}=$ área de poros com $C<0,2[15,18,24]$;

3. $A_{F m a x}>1,5=R D C l$ com $F_{\max }>1,5 \mathrm{~mm}[17-18]$;

4. $A_{p}>1000=R D C l$ com $P>1000 \mu m$;

5. $A c T-x=a . A D e q>300+b . A c<0,2+c . A F m a x>1,5+d . A p>1000$

onde, a,b,c e d são constantes.

A área crítica total para um dado ensaio $x$ (Аст- $\mathrm{x}$ ) é dada por uma equação, onde fatores de ponderamento indicam o peso da contribuição de cada tipo de área crítica. Esses fatores dependem, basicamente, do tipo de solicitação mecânica presente em cada ensaio e podem ser obtidos empiricamente. As Equações 4 e 5, descrevem as áreas críticas totais para os ensaios de tamboramento $\left(A_{C-1}\right)$ e compressão diametral (Ac-s).

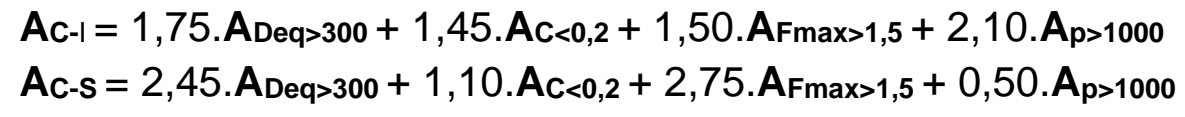

Uma vez que essas áreas críticas totais têm relação com o potencial de falha dos coques frente uma solicitação, pode-se utilizar tais áreas para prever os índices de resistência dos coques nos ensaios mecânicos. Para isso, foram realizadas regressões, a partir das Equações 4 e 5, encontrando as equações que melhor descrevem os índices de resistência mecânica (l600 e S) a partir das áreas críticas. (Figura 6a-b).

$$
\begin{aligned}
& \text { I } 600=98,9-0,337 . \text { Аст-I } \\
& \mathbf{S}=16,5-0,103 . \text { Аст-S }
\end{aligned}
$$

A partir dos valores (pesos) assumidos pelas constantes nas Equações 4 e 5 , podese notar que as áreas críticas das $\mathrm{RDCl}$, classificadas por perímetro $\left(A_{p}>1000\right)$, têm mais importância para as solicitações do ensaio de tamboramento (abrasão e queda), que no ensaio de compressão diametral (tração e compressão). O perímetro das RDCl está relacionado com efeitos de interface entre essas regiões com as RDCR. Esses resultados indicam que a qualidade das interfaces entre as regiões derivadas de componentes reativos e inertes, deve ter maior importância para as solicitações dinâmicas (tamboramento), que estáticas (compressão diametral). No entanto, a descrição de interface apenas pelo perímetro das RDCl é deficitária, uma vez que a qualidade das interfaces é distinta para cada tipo de inerte. A título de exemplo, as interfaces entre as regiões derivadas de compostos reativos e inertes do coque de petróleo são bem mais suaves que aquelas provenientes dos macerais da inertinita. Isso quer dizer que o desenvolvimento de um índice que exprima a qualidade das interfaces, provenientes de cada tipo de inerte, pode ser incorporado 
as relações de maneira a melhorar a descrição do comportamento mecânico dos coques nos ensaios dinâmicos.
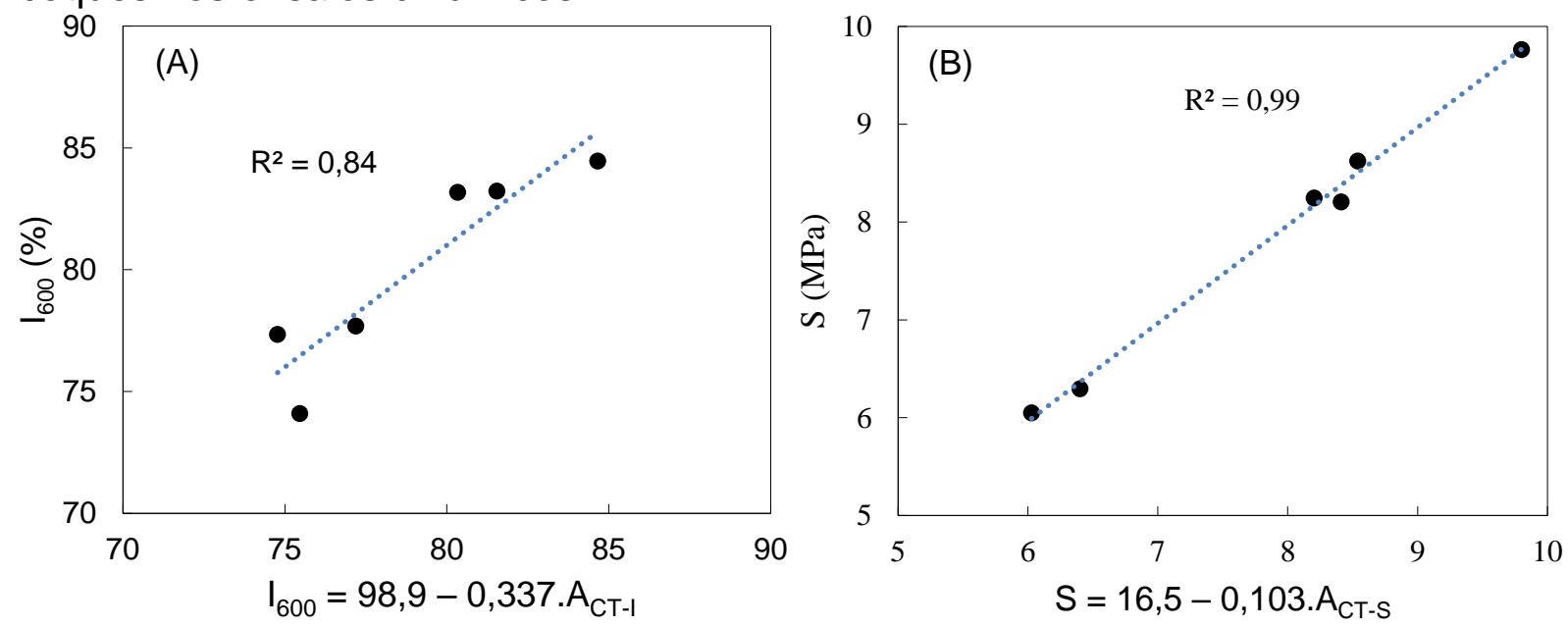

Figura 6. Relações entre os índices dos ensaios de resistência mecânica e as (a) Equação 6 - I 600 e (b) Equação 7 - S.

\section{CONCLUSÕES}

A microestrutura porosa dos coques estudados foi caracterizada pela associação de técnicas de microscopia ótica e análise de imagens, obtendo parâmetros referentes as RDCR e RDCl. A capacidade de descrição do comportamento mecânica dos coques através dos parâmetros microestruturais foi avaliada por dois grupos de modelos contidos na literatura: (1) relações entre parâmetros microestruturais médios dos coques e os índices mecânicos e (2) quantificação dos poros com características mais críticas a resistência mecânica.

Os resultados apresentados nesse trabalho mostraram que ambas abordagens não conseguem descrever o comportamento mecânico dos coques produzidos por carvões ricos em componentes inertes ( $>40 \%$ em volume). A esse resultado foi atribuído o fato que nenhuma das relações avaliadas contemplava parâmetros referentes as regiões derivadas de componentes inertes.

A partir disso foi sugerido uma nova relação incluindo tais parâmetros e critérios para classificação das RDCl como críticas. Ambas abordagens foram novamente avaliadas e observou-se significativa melhora nas correspondências das relações a partir da inclusão desses novos termos.

\section{Agradecimentos}

Os autores desse trabalho agradecem a Gerdau Usina Ouro Branco pelo fornecimento dos carvões importados, ao Conselho Nacional de Desenvolvimento Científico e Tecnológico (CNPq) pelo incentivo financeiro.

\section{REFERÊNCIAS}

1 AGRA, A.A. et. al. Microestrutura do coque: desenvolvimento de análise quantitativa via microscopia ótica associada a análise de imagem. In: 48ํㅗㄴ Seminário de Redução de Minérios e Matérias-Primas, ABM Week, São Paulo, SP. 2018.

2 AGRA, A.A. et. al. Coke microstructure - sample preparation and image analysis via ImageJ. In: $8^{\text {th }}$ International Council for Scientific and Technical Information (ICSTI), Schloss Schönbrunn, Apothekertrakt, Vienna, 2018. 
3 PUSZ, S. et. al. The dependence of physical structure of a coal heated in a coking chamber on non-uniform distribution of a temperature. In: Internacional Journal of Coal Geology. V. 82, p.125-131. 2010.

4 LOISON, R.; FOCH, P.; BOYER, A. Coke Quality and Production. 2nd ed. Butterworth \& Co, 1989.

5 SCHAPIRO, N.; GRAY, R.J.; EUSNER, G. Recent developments in coal petrography. Blast Furnace, Coke Oven and Raw Materials Committee, v. 20, p. 89-112. 1961.

6 PATRICK, J.W.; HANSON, S. Pore Structure of Graphite, Coke and Composites. In: Handbook of Porous Solids. V.3, p: 1900-1922. 2002.

7 EUDIER, M, Powder Metall, 9, 278 (1962). Tabor, D., In: Mechanical Properties of Non-Metallic Brittle Materials, ed. H. Walton, Butterworths, London, 1958.

8 MCADAM, G.D. The Mechanical Propertles of Cermets with a Metallic Matrix. Powder Met. V 10 (20), p. 307-17. 1967

9 NISHIOKA, K.; YOSHIDA, S. Strength Estimation of Coke as Porous Material. In: ISIJ, v. 23, p. 387-392. 1983.

10 HAYS, D.; PATRICK, J.W.; WALKER, A. An alternative approach to coke strength prediction. In: Fuel, V.68, p. 213-217; 1989.

11 PATRICK, J.W.; STACEY, A.E. The strength of industrial cokes: Part 6. Further studies of the influence of coke breeze in a coal charge on tensile strength of coke. In: Fuel, v 54, p. 256-264. 1975.

12 PATRICK, J.W. Microscopy of porosity in metallurgical cokes. In: Journal of Microscopy, v.132, p. 333-343. 1983.

13 PATRICK, J.W.; WALKER, A. Macroporosity in cokes: its significance, measurement, and control. In: Carbon, v. 27, p. 117-123. 1989.

14 ANDRIOPOULOS, N.; DUKINO, R.; SAKUROVS, R. The Strength controlling properties of coke and their relationship to tumble drum indices and coal type. In: Australian Coal Association Research Program. Final Report, 2002.

15 MENG, F. et. al. Characterization of microstructure and strength of coke particles and their dependence on coal properties. In: Powder Technology v320, p. 249-256. 2017.

16 KUBOTA, Y. et. al. Quantitative Evaluation of Relationship between Coke Strength and Pore Structure. In: ISIJ, v. 51, 1800-1808. 2011.

17 DONSKOI, E. et. al. Novel optical image analysis coke characterization and its application to study of the relationship between coke Structure, coke strength and parent coal composition. In: Fuel, v.208. p.281-295. 2017.

18 KUBOTA, Y. et. al. Effects of Coal Inertinite Size on Coke Strength. In: ISIJ. v. 48, p.563-571. 2008.

19 XING, X. et. al. Macrostrength and pore structure of coke subjected to gasification and anneling under blast furnace conditions. In: AISTech Conference Proceedings. p. 1-12. United States: AISTech. 2015.

20 BROWN, S.D.; BIDDULPH, R.B.; WILCOX, P.D. A Strength-Porosity Relation Involving Different Pore Geometry and Orientation. In: Journal of The American Ceramic Society. V. 47, p. 320-322. 1964.

21 BARRIOCANAL, C. et. al. The characterization of interfaces between textural components in metallurgical cokes. In: Fuel, v. 73, p. 1842-1847. 1994.

22 BARRIOCANAL, C. et. al. The quality of interfaces in metallurgical cokes containing petroleum coke. In: Fuel Processing Techonology. V.45, 1-10. 1995.

23 SAITO, Y. et. al. Image Recognition Method for Defect on Coke with Low-quality Coal. In: ISIJ, v.54, p. 2512-2518. 2014. 